Nota técnica

\title{
Estrategias para mejorar el aprendizaje de la biodiversidad amazónica en estudiantes
}

\section{[Strategies to improve learning about Amazonian biodiversity in students]}

\author{
José Lisbinio Cruz Guimaraes ${ }^{* 1,2,3}$, Rosana Gonzales Arzubialdes ${ }^{1}$, Eva María Panaifo Pinedo², \\ Julio César Paredes Gallo², Gabriel García Mendoza³
}

1. Universidad Científica del Perú (UCP), Av. Quiñones km 2,5, San Juan Bautista, Maynas, Loreto, Perú. Correo electrónico: josecruzguimaraes@gmail.com (J. Cruz. * Autor para correspondencia), rgonzales@ucp.edu.pe (R. Gonzales).

2. Programa de Educación Rural Fe y Alegría 47, Iquitos PERfyA 47. Av. Quiñones km 4,5, San Juan Bautista, Maynas, Loreto, Perú. Correo electrónico: evamariapanaifo@gmail.com (E. Panaifo), jgallito66@hotmail.com (J. Paredes). 3. Equipo Primatológico de Loreto EPL. Pasaje Internacional N²2, Belén, Maynas, Loreto, Perú.

Correo electrónico: garcia.mendoza25@gmail.com (G. Garcia).

\begin{abstract}
Resumen
Esta investigación está orientada a la aplicación de diferentes estrategias de Educación Ambiental como el desarrollo de sesiones de aprendizajes, actividades lúdicas y el uso de las Tecnologías de Información y Comunicación con la aplicación de materiales educativos interactivos de siete especies de flora y fauna; se trabajó con estudiantes de cuatro instituciones educativas del Programa de Educación Rural Fe y Alegría 47 del ámbito de la carretera Iquitos-Nauta, el año 2018, a fin de mejorar el conocimiento sobre la biodiversidad de la Amazonía. La muestra estuvo conformada por 128 estudiantes, seleccionados en forma no aleatoria por conveniencia; se empleó la encuesta como técnica de recolección de datos. Los resultados muestran que los estudiantes de las cuatro instituciones tuvieron un incremento significativo en la comprensión sobre la conservación y manejo de la biodiversidad amazónica, con el empleo de las siguientes estrategias: Software Educativo (TIC), Láminas Educativas y actividades lúdica de juegos de memoria imagen - imagen, posibilitando que los estudiantes sean capaces de construir su propio proceso de aprendizaje por medio de la experimentación, innovación, difusión y uso compartido de información y buenas prácticas. Finalmente se concluyen que la aplicación de estrategias en la educación ambiental mejora el aprendizaje de los estudiantes del Programa de Educación Rural Fe y Alegría 47 sobre los conocimientos biológicos y ecológicos de las especies de flora y fauna, por ende, se sugiere que estas estrategias podrían aplicarse en el programa educativo que reciben los estudiantes.
\end{abstract}

Palabras clave: Aprendizaje y tecnologías de información y comunicación (TIC), Educación ambiental, Estrategias pedagógicas, Diversidad biológica.

\begin{abstract}
This research was aimed at the application of different Environmental Education strategies in the rural area located on the Iquitos-Nauta road with students from the Fe y Alegría km47 Rural Education Program in 2018. We focused on the design and application of learning sessions and the use of Information and Communication Technologies with the application of interactive educational materials of seven species of flora and fauna to improve learning about the biodiversity of the Amazon. The sample consisted of 128 students, selected non-randomly for convenience. The students were surveyed for data collection as well as a questionnaire was used to collect data on the variables. The results show that the students of the four institutions had a significant increment in their understanding of the conservation and management of Amazonian biodiversity through the following strategies: Educational Software (ICT), Educational Plates and recreational activities of memory games image - image, enabling students to be able to build their own learning process through experimentation, innovation, dissemination and sharing of information and good practices. Finally, it is concluded that the application of environmental education strategies improves biological and ecological understanding in the students of the Fe y Alegría Rural Education Program 47. These strategies should be applied in the educational program that students receive less attention in the Peruvian Amazon.
\end{abstract}

Keywords: Biological diversity, Environmental education, Learning and information communication technologies (ICT), Pedagogical strategies. 


\section{INTRODUCCIÓN}

En la actualidad, existe un interés creciente sobre la educación ambiental y la protección del medio ambiente a nivel global (Conama, 2016). El Perú, no es ajeno a este interés ya que posee una gran tradición y diversidad natural y cultural, especialmente en materia biológica, geológica, climática y de saberes ancestrales (Ministerio de Educación y Ministerio del Ambiente, 2017). Sin embargo, así como el patrimonio natural abona al crecimiento económico de Perú (6,1\% promedio entre 2002 y 2013), se estima también que el costo económico de la degradación ambiental (equivalente en promedio al $4,1 \%$ del PBI nacional) reduce significativamente este crecimiento (Banco Mundial, 2013).

Para armonizar los aspectos ambientales con el crecimiento económico, la paz social y el bienestar general de la población, particularmente de las zonas rurales, se requiere el desarrollo e implementación eficaz de nuevas políticas públicas, con el propósito de mejorar los aprendizajes de los estudiantes en armonía con el medio ambiente. En este contexto el sistema educativo peruano tiene como uno de los ejes curriculares la Educación Ambiental (Ministerio del Ambiente, 2012), entendida como un proceso educativo integral que se da en toda la vida del individuo y que busca generar en éste las representaciones, emociones, disposiciones y modos de comportamiento necesarios para desarrollar sus actividades productivas o reproductivas en forma ambientalmente adecuada y contribuir al desarrollo sostenible del país (Muñoz, 2010). En este sentido la aplicación del enfoque ambiental se da a través del sistema educativo formal y no formal (Ministerio del Ambiente, 2013). Sin embargo, el aprendizaje de la gran diversidad biológica amazónica merece especial atención, por ser uno de las dificultades que tienen los estudiantes de la región Loreto, al no conocer la diversidad biológica amazónica en los diferentes niveles educativos, por las inadecuadas metodologías aplicadas, la carencia de medios y materiales educativos, y escasa motivación del estudiante en el aprendizaje de temas rela- cionados a la flora y fauna (IIAP, 2012). En tal sentido esta investigación se orienta en brindar herramientas, metodologías y técnicas que ayuden a mejorar la comprensión de la diversidad biológica amazónica y la calidad educativa ambiental, considerando el actual escenario en la que se desenvuelven los estudiantes y docentes. Asimismo, permitirá crear entornos de aprendizaje que mejoren la calidad y las oportunidades educativas en el marco del Proyecto Educativo Regional de Loreto (GOREL, 2010), facilitando el desarrollo de capacidades y logro de competencias exigidas por la sociedad actual y futura.

La investigación se desarrolló en las Instituciones Educativas Rurales dirigidas por Fe y Alegría 47, con estudiantes del nivel primario. La motivación para realizar este estudio fue la limitada inserción de temas amazónicos en los planes curriculares, debido a un escaso conocimiento de los estudiantes y docentes sobre las especies de la Amazonía peruana y su rol en el ecosistema (Ministerio del Ambiente, 2014), por esta razón se consideró importante desarrollar la investigación aplicando estrategias de educación ambiental para mejorar este aprendizaje. El trabajo se justifica en la necesidad de tener una mejor comprensión de la diversidad biológica amazónica y crear una actitud ambiental en los estudiantes del nivel primaria del Programa de Educación Rural Fe y Alegría 47 de la ciudad de Iquitos, lo que contribuirá a mejorar el cambio de actitud ambiental y promover la conservación de las especies y el desarrollo sostenible (SINIA, 1997). Los resultados obtenidos servirán como referencia para posteriores trabajos desde la implementación, ejecución de metodologías pertinentes y mejorar la calidad educativa y el conocimiento de la diversidad biológica, teniendo como objetivos: a) Sensibilizar a la comunidad educativa sobre la importancia de conservar la biodiversidad mediante charlas, talleres y b) aplicar estrategias de educación ambiental para valorar la biodiversidad. 


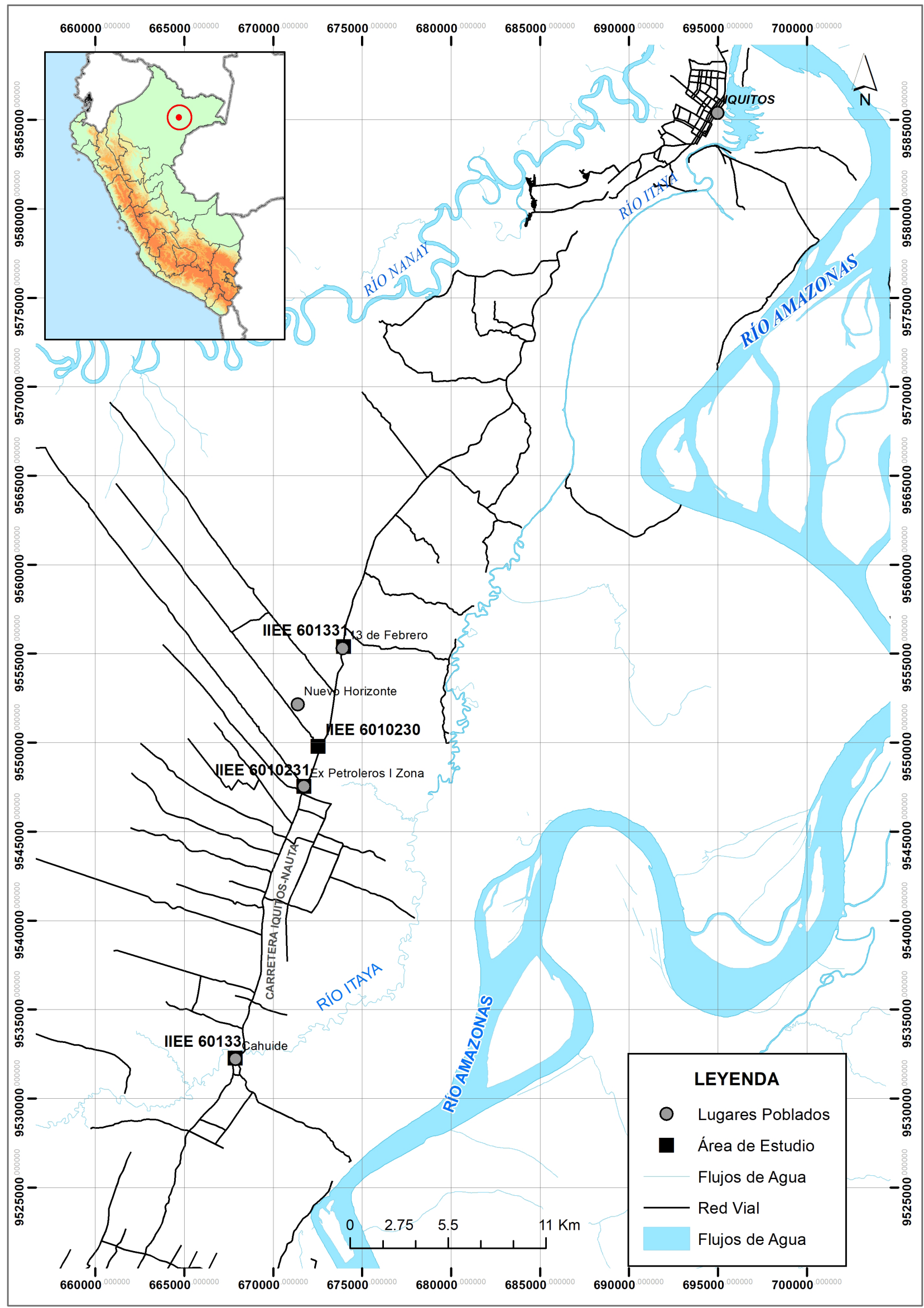

Figura 1. Ubicación del área de estudio, en el ámbito de la carretera Iquitos-Nauta. IIEE N 601331 CCPP 13 de febrero. IIEE Nº 6010230 CCPP Nuevo Horizonte. IIEE Nº 6010231 CCPP Ex Petroleros. Y el IIEE Nº 60133 CCPP Cahuide. 


\section{MATERIALES Y MÉTODO}

\section{Área de estudio}

La investigación se realizó en el ámbito de la carretera Iquitos - Nauta distrito de San Juan Bautista, provincia de Maynas, Perú (Figura 01). Se trabajó con cuatro instituciones educativas ubicados en el área de influencia de la carreta Iquitos - Nauta; IIEE № 601331 con 33 estudiantes, IIEE N 6010231 con 50 estudiantes, IIEE $N^{\circ} 6010230$ con estudiantes 13 e IIEE $N^{\circ}$ 60133 con 32 estudiantes respectivamente (Tabla 1).

\section{Diseño de investigación}

El diseño utilizado en el estudio fue el experimental del tipo pre experimento de pre prueba - post prueba con un solo grupo. Fue experimental, ya que hubo intervenciones sobre estrategias de educación ambiental como, por ejemplo: Software Educativo de biodiversidad amazónica, láminas con descripción didáctica de seis especies previamente elaboradas describiendo aspectos morfológicos, biológicos y ecológicos, actividades lúdicas de juegos de memoria imagen - imagen y ludo. se explicó aspectos biológicos y ecológicos de especies, como por ejemplo: Mauritia flexuosa ("aguaje"), Arapaima gigas ("paiche"), Tapirus terrestris ("sachavaca"), Pteronura brasiliensis ("lobo de río"), Trichechus inunguis ("manatí") y Osteoglossum bicihrrosum ("arahuana") y la importancia que cumplen en los ecosistemas. Fue pre-experimento de pre-prueba respecto a factores biológicos y ecológicos de las especies, post-prueba con un solo grupo porque su ejecución implica: Primero: una medida previa de la variable dependiente a ser estudiado en los sujetos (pre-prueba). Segundo: aplicación de la variable independiente a los sujetos del grupo. Tercero: una nueva medición de la variable dependiente en los sujetos (post-prueba).

\section{Técnicas de Recolección de Datos}

Las técnicas que se emplearon en la recolección de datos fueron: la encuesta porque se observó a las variables en forma indirecta. Los instrumentos de recolección de datos para ambas variables son: Variable Independiente $(X)$ : estrategias de educación ambiental y Variable Dependiente $(Y)$ : diversidad biológica amazónica; asimismo, se aplicó un cuestionario (pre-test y pos-test).

\section{Análisis de datos}

La población la conformaron todos los estudiantes del Programa de Educación Rural Fe y Alegría 47, de San Juan Bautista, fueron 230 entre niños y niñas. Y la muestra la conformaron los estudiantes de $6^{\circ}$ Grado de Educación Primaria del Programa de Educación Rural Fe y Alegría 47, que fueron: 128 niños. La selección de la muestra se hizo en forma no aleatoria por conveniencia. Se ha muestreado solo a estudiantes del sexto grado debido a que los estudiantes de cuarto y quinto grado de educación primaria, en zona rural tienen una baja comprensión lectora (MINEDU-UMC, 2018). El muestreo de o por conveniencia es una técnica no probabilística donde los sujetos son seleccionados dada la conveniente accesibilidad y proximidad de los sujetos para el investigador.

Esta investigación fue de tipo correlacional porque se midió el grado de correlación entre las variables: estrategias de educación ambiental y el aprendizaje sobre la diversidad biológica amazónica.

\section{RESULTADOS}

En la prueba de entrada se obtuvo los siguientes resultados, en las calificaciones en una escala de 0 a 20: rango: 5-11; promedio de calificaciones: 8; aprobados: 24 estudiantes; y desaprobados: 104. Mientras que luego de la sesión de aprendizaje los niños tuvieron las siguientes calificaciones: rango: 9-16; promedio 12; aprobados: 110; y desaprobado: 18 estudiantes.

La mejor estrategia empleada para incrementar el nivel de aprendizaje en niños resultó ser 
el uso del Software Educativo (promedio: 12) que Láminas Educativas (promedio de 7,25) y Actividades Lúdicas obtuvo (promedio de 5,75). Dichos materiales contienen texto, imágenes y vídeos; asimismo, tiene un lenguaje fácil y sencillo de acuerdo al nivel educativo.

Con estos resultados se logró conocer que la estrategia con mayor aceptación fue la utilización del Software Educativo de las especies de flora y fauna de la Amazonía en estudiantes del $6^{\circ}$ grado del nivel primario. Con estos resultados se logró el objetivo específico: Identificar el efecto de las estrategias de educación ambiental después de la aplicación del aprendizaje de la diversidad biológica amazónica. Entonces la aplicación de la estrategia de Educación Ambiental en los estudiantes ha sido favorable para su rendimiento académico, pues, ha contribuido al desarrollado de capacidades y habilidades con respecto a temas de flora y fauna amazónica.

Tabla 1. Distribución de Estudiantes por Institución Educativa.

\begin{tabular}{cc}
\hline IIEE & Cantidad de estudiantes \\
\hline 601331 & 33 \\
6010231 & 50 \\
6010230 & 13 \\
60133 & 32 \\
\hline TOTAL & $\mathbf{1 2 8}$ \\
\hline
\end{tabular}

\section{DISCUSIÓN}

El presente trabajo coincide con el reporte de Aline (2010), en el sentido que la inclusión en el programa de estudios de temas ambientales mejora considerablemente el conocimiento la diversidad biológica y el medioambiente que le rodea al estudiante, por otra parte, se logró un porcentaje alto (82\%) de aceptación superior al reportado por Aline (2010) de 40\%, probablemente se deba a que se empleó varias estrategias mientras que el citado autor solo uno. En nuestro trabajo, la estrategia Software Educativo obtuvo un $59 \%$ realizando la suma en las cuatro instituciones educativas, con un promedio $14 \%$ de aceptación y aprobados.
Los resultados obtenidos por Nascimento et al. (2007) empleando métodos lúdicos son coincidentes con los resultados obtenidos en el presente trabajo ya que fueron $18 \%$, lo que indica un método bastante eficaz para la sensibilización de la conservación de la fauna silvestre especialmente con especies de mayor representatividad en la Amazonía peruana.

También se ha empleado el software educativo como el "Multi-Ecológico" usado por Martins et al. (2008) para sensibilizar en los temas de contaminación ambiental; con resultados similares obtenidos en el presente trabajo empleando el Software Educativo de especies de flora y fauna de la Amazonía Peruana, que cuenta con efectos visuales y de sonidos con motivación lúdica, que permitieron sensibilizar a los estudiantes en pro de la conservación de las especies.

Mientras que, en pruebas realizadas sobre los conocimientos previos del medio ambiente, se encontró que los estudiantes conocen muy poco; similar resultado fue reportado por Santos et al. (2001), donde refieren una serie de instituciones como responsables de la educación ambiental por falta de una visión interdisciplinar y transversal, para incrementar el conocimiento sobre las especies de la diversidad biológica de la Amazonía. Por otro lado, los juegos electrónicos pueden ayudar bastante en la cultura de los estudiantes respecto al medio ambiente y la importancia del agua; entre otros, tal como refiere Ferreira et al. (2009), coincide plenamente con el presente trabajo donde también se empleó Tecnologías de Información (TIC), para concientización como una comunicación recreativa.

El juego como recurso didáctico en la enseñanza de la educación ambiental para estudiantes de nivel medio superior propuesto por Cabral (2012) es un método que fue corroborado durante la ejecución de la presente investigación porque permite diferentes actividades como juegos, pasatiempos, concursos, cantos, poemas etc., que hacen del aprendizaje algo agradable y dinámico.

Santos et al. (2011), realizaron estudios con estudiantes que ya habían tenido algún contacto 
con especies marinas sin embargo no fue suficiente los conocimientos adquiridos y que fue necesario un proceso de educación ambiental con lo cual se coincide ya que los resultados de la presente investigación también se corroboran lo afirmado por el autor antes citado al comprobar las pruebas de entrada y de salida con $77 \%$ y $93 \%$ de aprobados respectivamente.

Con la llegada de las tecnologías informáticas, el rol del docente está cambiando desde un enfoque centrado en el profesor - prácticas alrededor del pizarrón y el discurso, basado en clases magistrales - hacia una formación centrada principalmente en el alumno, dentro de un entorno interactivo de aprendizaje (UNESCO, 2004). En ese sentido, las Tecnologías de la Información y Comunicación (TIC) juegan un rol importante al proveer a los estudiantes la información y conocimientos necesarios para el proceso de enseñanza-aprendizaje.

Las instituciones educativas deben promover actividades permanentes orientadas a educar a los estudiantes promoviendo una cultura de conciencia ambiental que le permitan amar, conservar y defender la naturaleza de tal manera que pueda satisfacer sus necesidades fundamentales sin tener que perjudicar la biodiversidad.

\section{AGRADECIMIENTO}

Expresamos nuestro agradecimiento al Programa de Educación Rural Fe y Alegría 47 -Iquitos, por permitirnos realizar el trabajo de educación Ambiental.

\section{REFERENCIAS BIBLIOGRÁFICAS}

Aline, S. 2010. Educação Ambiental como parceria na educação tradicional: uma proposta de jogos ambientais - utilizando o lúdico e o pedagógico para a defesa do meio ambiente. Enciclopédia Biosfera, Goiânia, 6 (9), 1.

Banco Mundial 2013. Perú Panorama General.
Bernal, C. 2006. Metodología de la Investigación (2da Edición). México: Ed. Pearson.

Buendía, L.; Colás, B.; Hernández, P. 1998. Métodos de Investigación en Psicopedagogía. McGRAW-HILL / INTERAMERICANA DE ESPAÑA, S. A. U. Edificio Valrealty, 1.a planta. Madrid - España, 343 p.

Cabral, D. 2012. El juego como recurso didáctico en la enseñanza de la educación ambiental para estudiantes de nivel medio superior. Facultad de Estudios Superiores Iztacala.

Conama, 2016. Congreso Nacional del Medio Ambiente. Documento del Grupo de trabajo de Conama. GT - 19 La Respuesta Verde.

Ferreira, B.; Luciana, Diogo, G.; Zamboli, G. 2009. A Aplicabilidade de um Jogo Eletrônico na Educação Ambiental.

Gobierno Regional de Loreto GOREL 2010. Proyecto Educativo Regional PER.

Hernández, R. 2006. "Definición del alcance de la investigación a realizar: exploratoria, descriptiva, correlacional o explicativa" en Metodología de Investigación. México: McGraw- Hill, 57-68 pp.

Instituto de Investigaciones de la Amazonía Peruana IIAP. 2012. Programa de Investigación en Biodiversidad Amazónica PIBA. Capítulo II: Principales resultados de investigación.

Ley $N^{\circ}$ 26839. 1997. Ley sobre la conservación y aprovechamiento sostenible de la diversidad biológica, Publicado el 8 de julio.

Martins, G.; Nunes, M. A. y Viccari, R. M. 1998. Educação Ambiental suportada por um Ambiente de Ensino Inteligente. IV Congresso RIBIE, Brasilia, Instituto de Informática PUCRS.

Ministerio de educación, Ministerio del Ambiente. 2017. Plan Nacional de Educación Ambiental PLANEA.

Ministerio de Educación. 2018. Unidad de Medición de la Calidad de los Aprendizajes.

Ministerio del Ambiente. 2012. Informe de la Comisión Multisectorial encargada de elaborar propuestas normativas y políticas orientadas a mejorar condiciones ambientales y sociales en las que se desarrollan las actividades económicas, especialmente las 
industrias extractivas - Resolución Suprema $\mathrm{N}^{\circ}$ 189-2012-PCM.

Ministerio del Ambiente. 2013. Orientaciones para implementar la Política Nacional de Educación Ambiental a nivel multisectorial y descentralizado / Dirección General de Educación, Cultura y Ciudadanía Ambiental - Lima: Ministerio del Ambiente, 2013.

Ministerio del Ambiente. 2014. Especies de fauna silvestre peruana en los apéndices de la CITES. Actualización del Listado de Especies de Fauna Silvestre Peruana en los Apéndices de la CITES luego de la Decimosexta Reunión de la Conferencia de las Partes (CoP 16).

Muñoz, J. 2010. La educación ambiental como eje transversal del currículo. ISSN 19986047. Depósito Legal: GR 2922/2007 № 29 abril.
Nascimento, J.; Dessordi, A. Paola; De Souza, Daniele. 2007. produção de um jogo de ludo sobre a ariranha (Pteronura brasiliensis) para o ensino e divulgação da ecologia comportamental da espécie. Categoria Trabalho Acadêmico / Artigo Completo Eixo Temático - Educação Ambiental.

Otzen, T. y Manterola, C. 2017. Técnicas de muestreo sobre una población a estudio. Int. J. Morphol, 35 (1), 227-232.

Santos, F.; Nunes B.; Eugenia, C.; Lima, S.; Conceição, M.; Alves, H. 2011. Jogo Tartarugas: Objeto de Aprendizagem na Educação Ambiental. 1Universidade Federal de Sergipe - Cidade Universitária - CEP 49100000 - São Cristóvão - Sergipe - Brasil.

UNESCO. 2004. Las tecnologías de la información y la comunicación en la formación docente. Uruguay: Ediciones Trilce, 244 pp. 